\title{
Fibroblast growth factor receptor 4 Gly388Arg polymorphism associated with severity of gallstone disease in a Chinese population
}

\author{
Q. Chen ${ }^{1,2,3 *}$, W.J. Li ${ }^{2 *}$, Y.Y. Wan ${ }^{1}$, C.D. Yu² and W.G. Li ${ }^{1}$ \\ ${ }^{1}$ Department of Hepatobiliary Pancreas and Vessel Surgery, \\ Chenggong Hospital of Xiamen University, Xiamen, China \\ ${ }^{2}$ State Key Laboratory of Cellular Stress Biology, \\ School of Life Sciences, Xiamen University, Xiamen, China \\ ${ }^{3}$ The First Affiliated Hospital of Xiamen University, Xiamen, China \\ *These authors contributed equally to this study. \\ Corresponding author: W.G. Li \\ E-mail: lwg11861@163.com
}

Genet. Mol. Res. 11 (1): 548-555 (2012)

Received August 15, 2011

Accepted January 17, 2012

Published March 8, 2012

DOI http://dx.doi.org/10.4238/2012.March.8.3

\begin{abstract}
The etiology of gallstone disease is multifactorial; supersaturation of bile with cholesterol is a primary cause for gallstone formation. In previous studies, we found that fibroblast growth factor receptor 4 (FGFR4) plays an important role in maintaining bile acid homeostasis by regulating the expression of cholesterol $7 \alpha$-hydroxylase (CYP7A1), a rate-limiting enzyme for bile acid biosynthesis. The Gly388Arg (G-388R) polymorphism of FGFR4 affects stabilization and activation of FGFR4. Consequently, we studied the FGFR4 gene as a candidate gene for genetic susceptibility to gallstone disease. We found that overexpression of FGFR4, especially the G-388R mutant of FGFR4, inhibits luciferase activity of CYP7A1 reporter in HepG2 cells, indicating that the G-388R mutant of FGFR4 may have greater inhibitory activity against bile acid biosynthesis. To investigate the association of FGFR4 polymorphism with gallstone disease, 117 patients with gallstone
\end{abstract}


disease and 457 controls were genotyped for FGFR4 polymorphism G-388R by PCR-RFLP. Although the incidence of gallstone disease was not greater in patients with the FGFR4 RR genotype, the ratio of gallstone patients with acute cholecystitis in the FGFR4 RR genotype $(42 \%)$ was significantly higher than that in other genotypes of FGFR4 (P $=0.019)$. In conclusion, the $F G F R 4$ polymorphism is a genetic risk factor contributing to aggravation of gallstone disease.

Key words: Fibroblast growth factor receptor 4; Polymorphism; Gallstone disease

\section{INTRODUCTION}

Gallstone disease is a prevalent condition linked closely with cholecystitis and cholangiocarcinoma. It has become a major health problem worldwide (Lammert and Sauerbruch, 2005). Gallstone formation is characterized by abnormalities in cholesterol and bilirubin metabolism, and the main factor leading to the development of the disease is a cholesterol concentration in bile that exceeds the amount that will stay in solution (Johnston and Kaplan, 1993; Donovan, 1999). Bile acids are detergent molecules produced from cholesterol in the liver that are important for the solubilization of cholesterol in the gallbladder (Hylemon et al., 2009), so bile acids are involved in regulating cholesterol homeostasis.

The conversion of cholesterol to primary bile acids involves at least 14 distinct enzymes and is accomplished via 2 pathways: the neutral pathway and the alternative pathway. Cholesterol 7 $\alpha$-hydroxylase (CYP7A1), a cytochrome P450 enzyme located in the smooth endoplasmic reticulum of hepatocytes, is the limiting enzyme in the neutral pathway of bile acid biosynthesis (Russell and Setchell, 1992). Fibroblast growth factor receptor 4 (FGFR4) is a member of the FGFR family, which is activated by human FGF19 and its mouse ortho$\log$ FGF15 (Jones, 2008). FGFR4 knockout mice exhibit an elevated excretion of bile acids, bile acid pool size increase, and upregulation of CYP7A1 expression (Yu et al., 2000). Conversely, transgenic mice expressing a constitutively active form of FGFR4 display decreased CYP7A1 expression and reduced bile acid pool size (Yu et al., 2005). These studies implicate the FGF19/FGFR4 pathway in the regulation of bile acid homeostasis.

A genetic component in the susceptibility to gallstones was recognized as early as 1937 (Buch et al., 2007). Recent human genetic studies have indicated that genes involved in bile salt balance, including cholecystokinin A receptor and CYP7A1, are susceptibility genes for gallstone disease (Miyasaka et al., 2002; Jiang et al., 2004). Compared to the Gly388 variant, the Gly388Arg (G-388R) variation in the transmembrane domain (exon 9) of the FGFR4 protein results in FGFR4 stabilization and increasing phosphorylation after ligand binding (Wang et al., 2008). This variant has been associated with disease progression and survival in cancer patients (Wang et al., 2004; Falvella et al., 2009; Ye et al., 2010). However, the polymorphism of FGFR4 in patients with gallstone disease has never been studied.

In view of this background, we hypothesized that genetic variants of $F G F R 4$ may play a role in conferring genetic susceptibility to gallstone disease through regulation of CYP7A1. We investigated whether the FGFR4 polymorphism is an additional genetic risk factor contributing to the development and formation of gallstone disease. 


\section{PATIENTS AND METHODS}

\section{Patients and DNA isolation}

The study involved gallstone patients who underwent cholecystectomy in the Department of Hepatobiliary Pancreas and Vessel Surgery at the First Affiliated Hospital of Xiamen University. Healthy controls (blood donors) were recruited from the same hospital. Informed consent was obtained from each subject, and the study was approved by the Ethics Committee of the First Affiliated Hospital of Xiamen University. Genomic DNA of 117 patients (56\% females and $44 \%$ males; $49.5 \pm 14.4$ years) and 457 controls $(41 \%$ females and $59 \%$ males; $47.2 \pm 13.8$ aged years) was extracted from peripheral blood samples using a TIANamp Blood DNA Kit (TIANGEN, Beijing, China). Blood samples from all subjects were collected in vials containing ethylenediaminetetraacetic acid and stored at $-80^{\circ} \mathrm{C}$.

\section{Cell line and plasmids}

The human hepatocellular carcinoma cell line HepG2 was cultured in Dulbecco's modified Eagle's medium supplemented with $10 \%$ fetal bovine serum and $100 \mathrm{U} / \mathrm{mL}$ penicillin-streptomycin. The -443 to +54 fragment of the CYP7A1 promoter was inserted into the pGL3-basic vector to generate the CYP7A1 reporter plasmid. FGFR4 complementary DNA was cloned into the mammalian expression vector pCDNA3.1 to generate pCDNA3.1-FGFR4(Gly). The 388Arg mutant was constructed using a site-directed mutagenesis kit (Stratagene, La Jolla, CA, USA).

\section{Cell transfection and luciferase activity assay}

The cells were transfected with the CYP7A1 reporter plasmid together with pCR3.1-Rluc constitutively expressing Renilla luciferase as an internal control in the presence or absence of the pCDNA3.1-FGFR4(Gly) or pCDNA3.1-FGFR4(Arg) plasmids using a Lipofectamine 2000 (Invitrogen, Carlsbad, CA, USA) according to manufacturer instructions. Cells were harvested $24 \mathrm{~h}$ post-transfection, and luciferase activity was assayed and normalized to Renilla luciferase activity using a dual luciferase reporter assay system (Promega, Madison, WI, USA).

\section{Genotyping}

To determine the polymorphism of FGFR4 in patients and controls, we used polymerase chain reaction (PCR)-restriction fragment length polymorphism analysis. The target sequence containing the G-388R polymorphic site was amplified using a PCR procedure and the following primers: 5'-GACCGCAGCAGCGCCCGAGGCCAG-3' and 5'-AGAGGGAAGAGGGAGAGCTTCTG-3' (Bange et al., 2002). The $\mathrm{G}$ to R transition in codon 388 created a new $B s t$ NI restriction site. A 168-bp PCR product was digested with $B s t$ NI (TaKaRa, Dalian, China), and restriction fragments were analyzed on a $15 \%$ nondenaturing polyacrylamide gel visualized with ethidium bromide. 


\section{Statistical analysis}

Differences in genotype frequencies between the two groups studied were estimated using the chi-square test and were tested for conformity to Hardy-Weinberg equilibrium. Binary logistic regression was used for all analysis variables to estimate risk as odds ratio (OR) with $95 \%$ confidence intervals $(95 \% \mathrm{CI})$. Differences in bilirubin concentration for patients among genotypes were calculated using one-way analysis of variance. Tests of statistical significance in the transfection experiment were analyzed with a two-tailed Student $t$-test. All statistical analyses were performed using the SPSS 13.0 statistical software package (SPSS, Chicago, IL, USA). P values less than 0.05 were considered to be statistically significant.

\section{RESULTS}

\section{Effect of FGFR4 G-388R polymorphism on the expression of CYP7A1}

Arginine replacing glycine at amino acid 388 of FGFR4 is a common polymorphism of FGFR4, and the 388Arg mutant results in increased receptor stability and sustained receptor activation (Wang et al., 2008). To determine whether the polymorphism of FGFR4 affects the activation of FGFR4, we investigated the effects of the two genotypes of FGFR4 on the expression of CYP7A1 using a CYP7A1 promoter luciferase reporter. As shown in Figure 1, CYP7A1 promoter luciferase activity was significantly decreased in HepG2 cells transfected with the 388Gly genotype of FGFR4 expression vector $(\mathrm{P}=0.003)$, and the 388Arg mutant enhanced the activity of FGFR4 $(\mathrm{P}=0.011)$. The results indicated that the 388Arg mutation of FGFR4 enhanced the inhibitory effect of FGFR4 on CYP7A1 expression, and this polymorphism may be associated with the development or progression of gallstone disease.

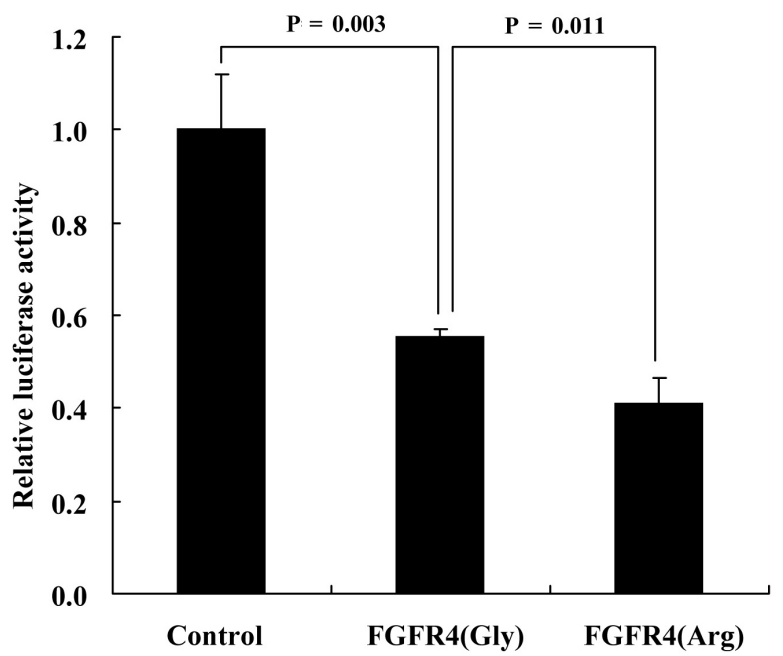

Figure 1. Effect of 388Gly or 388Arg genotype of FGFR4 on CYP7A1 expression. HepG2 cells were transfected with CYP7A1 reporter plasmid together with pCDNA3.1-FGFR4(Gly) or pCDNA3.1-FGFR4(Arg) and the luciferase activity was measured $24 \mathrm{~h}$ post-transfection. The pCDNA3.1 vector was the control. Data are reported as means $+\mathrm{SD}(\mathrm{N}=3)$. 


\section{Genotype frequencies of FGFR4 G-388R polymorphisms and gallstone disease}

The FGFR4 G-388R polymorphism distributions of gallstone patients and healthy controls are shown in Table 1. The study comprised 117 patients with gallstone disease and 457 healthy volunteers. Among the 117 gallstone patients, 41 (35.04\%) were homozygous for the $\mathrm{G}$ allele; 57 were heterozygous (48.72\%) and 19 homozygous (16.24\%) for the R allele. The distribution frequency of the FGFR 4 genotype in gallstone patients was not significantly different from that in healthy subjects $(\mathrm{P}=0.350)$. A multiple variable logistic regression analysis showed that the ORs for the 388GR and 388RR genotype were $0.807(95 \% \mathrm{CI}=$ $0.512-1.272)$ and $0.649(95 \% \mathrm{CI}=0.355-1.187)$, respectively; the $\mathrm{R}$ allele also displayed no significant difference at the allele level $(\mathrm{OR}=0.807,95 \% \mathrm{CI}=0.603-1.081$; Table 2).

The traditional risk factors for gallstone disease are the four $F_{\mathrm{s}}$ : female, fat, 40 , and fertile; therefore, the FGFR 4 polymorphism distribution was further analyzed according to age and gender. As shown in Table 1, in the age- and gender-stratified analyses, 3 genotype frequencies of the FGFR 4 gene in gallstone patients were not significantly different from those in healthy controls. These results demonstrate that the G-388R mutation of the FGFR4 gene does not contribute to the genesis of gallstone disease.

\begin{tabular}{|c|c|c|c|c|c|c|c|}
\hline \multirow[t]{2}{*}{$\overline{\text { Variable }}$} & \multicolumn{3}{|c|}{ Patients } & \multicolumn{3}{|c|}{ Controls } & \multirow[t]{2}{*}{$P$ values ${ }^{1}$} \\
\hline & GG & GR & $\mathrm{RR}$ & GG & GR & $\mathrm{RR}$ & \\
\hline Total & $41(35.0)$ & $57(48.7)$ & $19(16.2)$ & $133(29.1)$ & $229(50.1)$ & $95(20.8)$ & 0.350 \\
\hline$\leq 40$ & $13(48.2)$ & $10(37.0)$ & $4(14.8)$ & $50(31.7)$ & $81(51.3)$ & $27(17.1)$ & 0.237 \\
\hline$>40$ & $28(31.1)$ & $47(52.2)$ & 15 (16.7) & $83(27.8)$ & $148(49.5)$ & $68(22.7)$ & 0.442 \\
\hline \multicolumn{8}{|l|}{ Gender } \\
\hline Male & $20(39.2)$ & $26(51.0)$ & $5(9.8)$ & $72(26.9)$ & $142(53.0)$ & $54(20.2)$ & 0.081 \\
\hline Female & $21(31.8)$ & $31(47.0)$ & $14(21.2)$ & $61(32.3)$ & $87(46.0)$ & $41(21.7)$ & 0.991 \\
\hline
\end{tabular}

Data are reported as number with percent in parentheses. ${ }^{1}$ Genotype frequencies of FGFR4 G-388R between gallstone patients and healthy controls were analyzed by the chi-square test and the $\mathrm{P}$ values represent a genotypic association test.

Table 2. Regression analysis of the polymorphism of FGFR4 G-388R in gallstone patients and healthy controls.

\begin{tabular}{lcccc}
\hline Genotypes/alleles & Patients (\%) & Controls (\%) & P values & OR (95\%CI) \\
\hline GG & 35.04 & 29.10 & - & 1 (reference) \\
GR & 48.72 & 50.11 & 0.358 & $0.807(0.512-1.272)$ \\
RR & 16.24 & 20.79 & 0.154 & $0.649(0.355-1.187)$ \\
G & 59.40 & 54.16 & - & 1 (reference) \\
R & 40.60 & 45.84 & 0.149 & $0.807(0.603-1.081)$ \\
\hline
\end{tabular}

$\mathrm{OR}=$ odds ratio $; \mathrm{CI}=$ confidence interval.

\section{Association of the FGFR4 G-388R polymorphism in gallstone patients with acute cholecystitis}

Gallstone disease may be divided into three clinical stages: asymptomatic, symptomatic, and complicated. Acute cholecystitis, the most common complication of gallstone disease, is a chemical inflammation usually requiring cystic duct obstruction and supersaturated bile (Strasberg, 1997). We analyzed the ratio of gallstone patients with acute cholecystitis to 
those without this symptom in various FGFR4 G-388R genotypes. As shown in Table 3, the ratio was significantly higher in the RR genotype $(\mathrm{P}=0.019)$. The results demonstrate that the G-388R mutation of the FGFR4 gene might be associated with the stages of gallstone disease.

Table 3. The ratio of patients with acute cholecystitis grouped by the polymorphism of FGFR4 G-388R.

\begin{tabular}{lccc}
\hline Genotypes & Patients with acute cholecystitis (\%) & P values & OR (95\%CI) \\
\hline GG & 14.63 & - & 1 (reference) \\
GR & 22.81 & 0.313 & $1.723(0.595-4.996)$ \\
RR & 42.11 & 0.019 & $4.242(1.207-14.908)$ \\
\hline
\end{tabular}

$\mathrm{OR}=$ odds ratio; $\mathrm{CI}=$ confidence interval.

Levels of serum bilirubin are significantly elevated in gallstone disease. A recent study has shown that total bilirubin is a useful predictor of persisting common bile duct stones in gallstone pancreatitis (Chan et al., 2008). Therefore, we further analyzed the levels of direct, neonatal, and total bilirubin in various genotypes of gallstone patients. As shown in Figure 2, mean serum total bilirubin, direct bilirubin, and neonatal bilirubin values were slightly higher in patients with the $388 \mathrm{R}$ mutation of FGFR 4 but were not significantly different compared with the GG and GR genotypes.

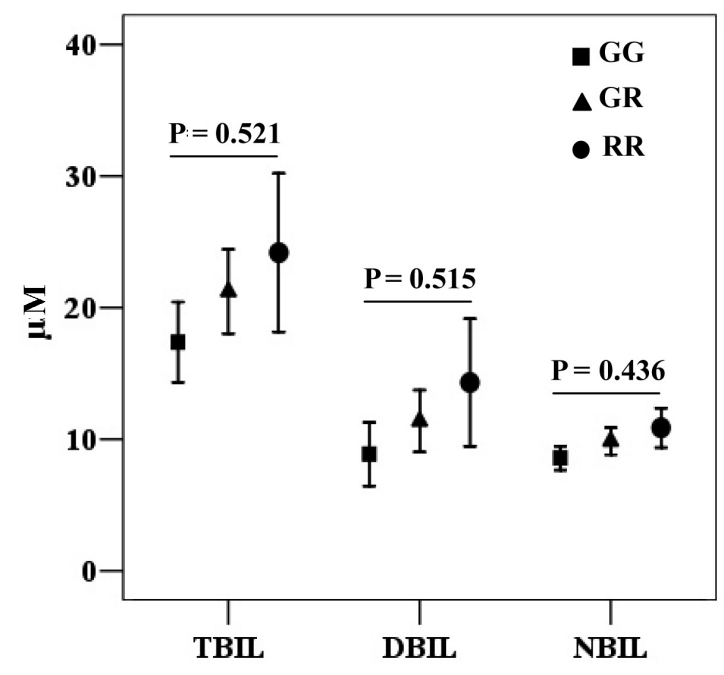

Figure 2. Effect of $388 \mathrm{R}$ mutation of $F G F R 4$ on serum bilirubin levels in the gallstone population. The difference among bilirubin levels was evaluated for statistical significance using one-way analysis of variance. TBIL $=$ total bilirubin; $\mathrm{DBIL}=$ direct bilirubin; NBIL = neonatal bilirubin .

\section{DISCUSSION}

Gallstones form as the culmination of a complex series of events that result in the precipitation of insoluble substances in the gallbladder, cholesterol hypersecretion, supersaturation of calcium carbonate, impaired motility of the gallbladder, and so on (Johnston and Kaplan, 1993; Donovan, 1999). FGFR4 has been reported to regulate bile acid homeostasis and gallbladder filling in mice (Yu et al., 2000, 2005; Choi et al., 2006). FGFR4 G-388R 
polymorphism, one of the most frequent polymorphisms, results in FGFR4 stabilization and increased phosphorylation after ligand binding (Wang et al., 2008). Therefore, our preliminary hypothesis was that FGFR4 G-388R polymorphism might affect the formation of gallstones through the regulation of bile acid homeostasis and gallbladder motility.

Our results showed that although this polymorphism enhances the inhibition of CYP7A1 expression by FGFR4, it did not contribute to gallstone formation. Likewise, A-204C polymorphism of CYP7A1 was not associated with gallstone disease in our study (data not shown). A previous study has shown that the A allele of CYP7A1 might be considered a risk gene for gallstone disease (Jiang et al., 2004), but another group showed that the CC genotype of CYP7A1 conferred a marginally significant risk for gallstone disease (Srivastava et al., 2008). These results suggest that the polymorphism of genes related to bile acid homeostasis, including CYP7A1 and FGFR4, might have a role in gallstone formation, but the population studied could also explain the observed effect.

Most patients with gallstone disease (75-80\%) remain asymptomatic (Strasberg and Clavien, 1992). We found that $42 \%$ of gallstone patients who exhibited acute cholecystitis were in the RR genotype of FGFR4, indicating that although the $388 \mathrm{R}$ mutation does not contribute to gallstone formation, it might aggravate disease progression in patients with gallstones. Owing to the influence of this polymorphism on bile acid synthesis, the resulting deficiency of the bile acid pool may lead to the accumulation of cholesterol in the gallbladder, which may aggravate gallstone disease. A recent study has reported that bilirubin level was associated with gallstone risk (Buch et al., 2010), but our results showed that FGFR4 G-388R polymorphism had no association with the level of bilirubin. Therefore, the mechanism for this phenotype requires further investigations.

In conclusion, this study is the first to describe a genetic relationship between FGFR4 polymorphism and gallstone disease. Further study will be required to provide supporting evidence for the involvement of the 388R mutation of FGFR4 in the aggravation of gallstone disease.

\section{ACKNOWLEDGMENTS}

Research supported by grants from the Natural Science Foundation of Fujian Province of China (\#2008J0110, \#2009D014, and \#2010D015), the China Postdoctoral Science Foundation (\#20100480713), and the Youth Foundation of the Health Department of Fujian Province (\#2010-2-84).

\section{REFERENCES}

Bange J, Prechtl D, Cheburkin Y, Specht K, et al. (2002). Cancer progression and tumor cell motility are associated with the FGFR4 $\mathrm{Arg}^{388}$ allele. Cancer Res. 62: 840-847.

Buch S, Schafmayer C, Volzke H, Becker C, et al. (2007). A genome-wide association scan identifies the hepatic cholesterol transporter ABCG8 as a susceptibility factor for human gallstone disease. Nat. Genet. 39: 995-999.

Buch S, Schafmayer C, Volzke H, Seeger M, et al. (2010). Loci from a genome-wide analysis of bilirubin levels are associated with gallstone risk and composition. Gastroenterology 139: 1942-1951.

Chan T, Yaghoubian A, Rosing D, Lee E, et al. (2008). Total bilirubin is a useful predictor of persisting common bile duct stone in gallstone pancreatitis. Am. Surg. 74: 977-980.

Choi M, Moschetta A, Bookout AL, Peng L, et al. (2006). Identification of a hormonal basis for gallbladder filling. Nat. Med. 12: 1253-1255.

Donovan JM (1999). Physical and metabolic factors in gallstone pathogenesis. Gastroenterol. Clin. North Am. 28: 75-97. Falvella FS, Frullanti E, Galvan A, Spinola M, et al. (2009). FGFR4 Gly388Arg polymorphism may affect the clinical 
stage of patients with lung cancer by modulating the transcriptional profile of normal lung. Int. J. Cancer 124: 28802885.

Hylemon PB, Zhou H, Pandak WM, Ren S, et al. (2009). Bile acids as regulatory molecules. J. Lipid Res. 50: 1509-1520. Jiang ZY, Han TQ, Suo GJ, Feng DX, et al. (2004). Polymorphisms at cholesterol 7alpha-hydroxylase, apolipoproteins B and $\mathrm{E}$ and low density lipoprotein receptor genes in patients with gallbladder stone disease. World J. Gastroenterol. 10: $1508-1512$.

Johnston DE and Kaplan MM (1993). Pathogenesis and treatment of gallstones. N. Engl. J. Med. 328: 412-421.

Jones S (2008). Mini-review: endocrine actions of fibroblast growth factor 19. Mol. Pharm. 5: 42-48.

Lammert F and Sauerbruch T (2005). Mechanisms of disease: the genetic epidemiology of gallbladder stones. Nat. Clin. Pract. Gastroenterol. Hepatol. 2: 423-433.

Miyasaka K, Takata Y and Funakoshi A (2002). Association of cholecystokinin A receptor gene polymorphism with cholelithiasis and the molecular mechanisms of this polymorphism. J. Gastroenterol. 37 (Suppl 14): 102-106.

Russell DW and Setchell KD (1992). Bile acid biosynthesis. Biochemistry 31: 4737-4749.

Srivastava A, Pandey SN, Choudhuri G and Mittal B (2008). Role of genetic variant A-204C of cholesterol 7alphahydroxylase (CYP7A1) in susceptibility to gallbladder cancer. Mol. Genet. Metab. 94: 83-89.

Strasberg SM (1997). Cholelithiasis and acute cholecystitis. Baillieres Clin. Gastroenterol. 11: 643-661.

Strasberg SM and Clavien PA (1992). Cholecystolithiasis: lithotherapy for the 1990s. Hepatology 16: 820-839.

Wang J, Stockton DW and Ittmann M (2004). The fibroblast growth factor receptor-4 Arg388 allele is associated with prostate cancer initiation and progression. Clin. Cancer Res. 10: 6169-6178.

Wang J, Yu W, Cai Y, Ren C, et al. (2008). Altered fibroblast growth factor receptor 4 stability promotes prostate cancer progression. Neoplasia 10: 847-856.

Ye Y, Shi Y, Zhou Y, Du C, et al. (2010). The fibroblast growth factor receptor-4 Arg388 allele is associated with gastric cancer progression. Ann. Surg. Oncol. 17: 3354-3361.

Yu C, Wang F, Kan M, Jin C, et al. (2000). Elevated cholesterol metabolism and bile acid synthesis in mice lacking membrane tyrosine kinase receptor FGFR4. J. Biol. Chem. 275: 15482-15489.

Yu C, Wang F, Jin C, Huang X, et al. (2005). Independent repression of bile acid synthesis and activation of c-Jun N-terminal kinase (JNK) by activated hepatocyte fibroblast growth factor receptor 4 (FGFR4) and bile acids. J. Biol. Chem. 280: 17707-17714. 